\title{
"Slow Activity Transients" in Infant Rat Visual Cortex: A Spreading Synchronous Oscillation Patterned by Retinal Waves
}

\author{
Matthew T. Colonnese and Rustem Khazipov \\ Institut de Neurobiology de la Méditérannée, Inserm, Unité 901, 13273 Marseille cedex 09, France
}

A primary feature of the preterm infant electroencephalogram is the presence of large infra-slow potentials containing rapid oscillations called slow activity transients (SATs). Such activity has not been described in animal models, and their generative mechanisms are unknown. Here we use direct-current and multisite extracellular, as well as whole-cell, recording in vivo to demonstrate the existence of regularly repeating SATs in the visual cortex of infant rats before eye opening. Present only in absence of anesthesia, SATs at postnatal day $10-11$ were identifiable as a separate group of long-duration $(\sim 10 \mathrm{~s})$ events that consisted of large $(>1 \mathrm{mV})$ negative local-field potentials produced by the summation of multiple bursts of rapid oscillatory activity $(15-30 \mathrm{~Hz})$. SATs synchronized the vast majority of neuronal activity (87\%) in the visual cortex before eye opening. Enucleation eliminated SATs, and their duration, interevent interval, and sub-burst structure matched those of phase III retinal waves recorded in vitro. Retinal waves, however, lacked rapid oscillations, suggesting that they arise centrally. Multielectrode recordings showed that SATs spread horizontally in cortex and synchronize activity at coactive locales via the rapid oscillations. SATs were clearly different from ongoing cortical activity, which was observable as a separate class of short bursts from postnatal day 9. Together, our data suggest that, in vivo, early cortical activity is primarily determined by peripheral inputs - retinal waves in visual cortex - that provide excitatory input, and by thalamocortical circuitry, which transforms this input to beta oscillations. We propose that the synchronous oscillations of SATs participate in the formation of visual circuitry.

\section{Introduction}

Correlated spontaneous neuronal activity regulates a number of developmental processes, including the formation, retention, and elimination of synapses, as well as proper connectivity and map formation (Zhou et al., 2003; Moody and Bosma, 2005; Colonnese and Constantine-Paton, 2006; Ben-Ari et al., 2007; Huberman et al., 2008). Consequently, a number of unique patterns of network activity are observed during early development (Khazipov and Luhmann, 2006). In sensory cortical areas, the most robust network activity observed in rats during the first postnatal week are "spindle bursts," a 0.5-3 s burst of rhythmic, alpha-beta $(5-30 \mathrm{~Hz})$ activity that is triggered in somatosensory cortex (S1) by spontaneous myoclonic twitches and in visual cortex (V1) by phase II retinal waves (Khazipov et al., 2004; Hanganu et al., 2006; Yang et al., 2009). Electroencephalographic (EEG) studies in human preterm infants reveal a homologous pattern, the "delta brush," that occurs spontaneously but also, like spindle bursts, in response to spontaneous myoclonic twitches (Milh et al., 2007).

\footnotetext{
Received 0ct. 7, 2009; revised Feb. 3, 2010; accepted Feb. 18, 2010.

This work was supported by Agence Nationale de la Recherche and Fondation pour la Recherche Médicale grants (R.K.) and National Eye Institute/National Institutes of Health Grant EY016966 (M.T.C.).

Correspondence should be addressed to Matthew T. Colonnese, Institut de Neurobiology de la Méditérannée, Parc Scientifique de Luminy, Boîte Postale 13, 13273 Marseille cedex 09, France. E-mail: colonnese@inmed. univ-mrs.fr.

DOI:10.1523/JNEUROSCI.4995-09.2010

Copyright $\odot 2010$ the authors $\quad 0270-6474 / 10 / 304325-13 \$ 15.00 / 0$
}

Recent advances using direct-coupled (DC) EEG recordings in preterm infants have demonstrated the existence of developmentally regulated slow activity transients (SATs), which organize most of activity in premature human neonates in temporal and occipital regions (Vanhatalo et al., 2005; Tolonen et al., 2007). SATs are multiband events, characterized by a large (up to $700 \mu \mathrm{V})$, infra-slow $(<0.5 \mathrm{~Hz})$ negative wave that nests activity at several higher-frequency bands. Currently, there is no animal model of SATs, and the mechanistic basis of this critical network activity is unknown. Several long-duration activity patterns occurring in immature cortex suggest two different models for SATs. A cortical generator is suggested by the existence of longduration migrating calcium waves and electrical oscillations identified in isolated cortical slices (Garaschuk et al., 2000; Dupont et al., 2006; Allène et al., 2008) and verified in vivo (Adelsberger et al., 2005; Yang et al., 2009). Conversely, longduration, multifrequency "mega bursts" occurring before eye opening in ferret visual cortex in vivo are eliminated by enucleation (Chiu and Weliky, 2001), suggesting that such activity is dependent on peripheral input, in this case, spontaneous retinal waves. Furthermore, the similar oscillatory structure of SATs and spindle bursts suggests a common generative mechanism dependent on peripheral stimulation, but, to date, infra-slow potentials and long-duration activity similar to SATs have not been observed in the rat.

To identify and study SATs, we used DC recordings of spontaneous activity in the visual cortex of unanesthetized rats before eye 
opening. We show the development of long-duration infra-slow waves containing rapid oscillations during the second postnatal week that match the description of human SATs. Generation of SATs in visual cortex results from the long-lasting excitation provided by phase III retinal waves (Kerschensteiner and Wong, 2008; Blankenship et al., 2009) but also from the thalamocortical circuitry that converts this input into highly periodic beta oscillations that synchronize cortical activity.

\section{Materials and Methods}

Animal care. All experiments were performed in accordance with Inserm and National Institutes of Health guidelines for the care of animals in research and were approved by the local review committee. Postnatal day 0 (P0) was the day of birth, and $90 \%$ of the rats opened their eyes between $\mathrm{P} 13$ and P14. The majority of experiments were performed with Wistar rats for correspondence with previous experiments. Albinism is associated with a reduction in visual acuity (Prusky et al., 2002) and an increased crossing of retinal ganglion cell axons (Lund, 1965). In light of this, we recorded from the monocular region of visual cortex and limited our analysis to the earliest visual development. Cortical activity in nine Long-Evans rats examined at the critical P9-P11 time point could not be differentiated from that of Wistar rats. These rats were used for the experiments reported in Figures $4 D$ and $5 C$. Recording techniques have been extensively presented (Hanganu et al., 2006; Minlebaev et al., 2007, 2009). In brief, head-fixed rat pups were prepared for extracellular recording under isoflurane anesthesia (2-3\% depending on age and verified by toe pinch). Xylocaine analgesia was applied to all cut surfaces and buprenorphine $(0.02 \mathrm{mg} / \mathrm{kg}$, i.p.) was given to manage pain. Recordings were made $1 \mathrm{~h}$ after recovery from anesthesia. Pups were kept restrained for $<5 \mathrm{~h}$ and were closely monitored for signs of stress and to ensure that they spent a normal proportion of their time sleeping. Body temperature was maintained between 35 and $36^{\circ} \mathrm{C}$. The animals were kept in low-light conditions throughout all experiments. For enucleation experiments, the animals were reanesthetized with $2-3 \%$ isoflurane in the recording setup, and the eye was removed. Local anesthetic (2\% xylocaine) was applied to the wound, and $10 \mathrm{~min}$ was allowed before removal of anesthesia. For eye injection, the eyelid was opened during the initial surgery. Before injection, xylocaine (1\%) in PBS was applied to the eyeball every $20 \mathrm{~min}$. A puncture was made at the ciliary margin, a Hamilton syringe needle (30 gauge) was inserted into the vitreous, and solution was injected $(5-10 \mu \mathrm{l})$.

Electrophysiological recordings and analysis. Recordings were made either with pulled glass microelectrodes (1-2 M $\Omega$ ) coupled to a directcurrent amplifier (Molecular Devices) or multisite linear array "Michigan probes" (NeuroNexus Technologies) coupled to a custom-built alternating current amplifier (1000×; bandpass, $1 \mathrm{~Hz}$ to $5 \mathrm{kHz}$ ). V1 recordings were localized at $2.8-3.2 \mathrm{~mm}$ lateral to midline and $0.0-0.5$ $\mathrm{mm}$ rostral to lambda. Electrode location was verified postmortem via tissue damage (glass electrodes) or dye localization (silicon probes). In animals older than P8, the electrode was further confirmed to be in V1 via monitoring of the response to $100 \mathrm{~ms}$ light flashes (M.T.C., unpublished data). Frequencies below $1-2 \mathrm{~Hz}$ were poorly transmitted by the Michigan probes and our amplifier; therefore, all multielectrode recordings were high-pass filtered at $2 \mathrm{~Hz}$. All recordings were amplified $1000 \times$, recorded in Axoscope, and analyzed with Clampfit (Molecular Devices) and Matlab (MathWorks).

Layer identification was accomplished via multiple criteria. Anatomical sections show layer 4 to be located $300-500 \mu \mathrm{m}$ below the pial surface. As shown here and by others, current sinks to thalamic input are maximal at the layer 3-4 border at these ages (Molnár et al., 2003). In almost all animals older than P8, we observed large units with a high rate of tonic spontaneous activity between 500 and $600 \mu \mathrm{m}$ depth, previously identified as layer 5a (Le Bon-Jego and Yuste, 2007). For purposes of this study, single electrodes were placed in layer 4 by locating the maximal fieldpotential deflection for the fast oscillations $300-500 \mu \mathrm{m}$ below the pial surface. For multielectrode recordings, the electrode was lowered until the topmost contact just touched the cortical surface. Layer 4 was defined as the electrode $300-500 \mu \mathrm{m}$ deep that was also $100-200 \mu \mathrm{m}$ above presumptive layer $5 \mathrm{a}$. Layers $2 / 3$ were defined as the electrodes $>100 \mu \mathrm{m}$ from the pial surface and separated by one electrode from layer 4 (i.e., we left a "buffer" electrode). Layers 5/6 were the remaining electrodes below $5 \mathrm{a}$ and above $1000 \mu \mathrm{m}$ depth. Electrodes were labeled with DiI, and the electrode tracks were confirmed to be in V1 by postmortem analysis. Multiunit firing was identified by high-pass filtering above $300 \mathrm{~Hz}$ and simple threshold discrimination ( $>4.3$ times SD of baseline noise). Good discrimination was verified for each channel.

Blind whole-cell patch-clamp recordings were performed using an Axopatch 200A amplifier (Molecular Devices) using an in vivo patching technique similar to that described previously (Khazipov et al., 2004). The pipettes were filled with the following solution (in mM): $135 \mathrm{Cs}-$ gluconate, $2 \mathrm{MgCl}_{2}, 0.1 \mathrm{CaCl}_{2}, 1$ EGTA, and 10 HEPES, pH 7.25. Membrane potential values were corrected for liquid junction potential of $+12 \mathrm{mV}$ and series resistance.

For retinal recordings, retinas were acutely dissected in ice-cold oxygenated artificial CSF (ACSF) (in mm: $126 \mathrm{NaCl}, 3.5 \mathrm{KCl}, 1.2 \mathrm{NaH}_{2} \mathrm{PO}_{4}$, $26 \mathrm{NaHCO}_{3}, 1.3 \mathrm{MgCl}_{2}, 2.0 \mathrm{CaCl}_{2}$, and 10 D-glucose, $\mathrm{pH} 7.4$ ) under red light. For recording, a retina was placed ganglion cell layer up and continuously perfused $(3 \mathrm{ml} / \mathrm{min})$ with $34^{\circ} \mathrm{C}$ ACSF in the dark. Insulated nivachrome wire $(50 \mu \mathrm{m})$ electrodes were lightly touched to the surface to record action potentials. Between one and four large-amplitude units could be distinguished at each location.

Multielectrode recordings. Ten SATs were manually selected from each of eight P10-P11 pups, each with a duration 8-10 s and clear beta oscillations, and confirmed to have unit activity on each channel. For troughtriggered averaging, all layer 4 events with a negative deflection $>75 \mu \mathrm{V}$ and lasting $<10 \mathrm{~ms}$ were automatically detected. The current source density (CSD) of the average current was calculated for each animal using the technique of Mitzdorf (1985). An average spike rate was also calculated and normalized to the peak spike rate for that pup. Population averages were calculated from the animal averages $(n=8)$. Crosscorrelation analysis was performed for each animal using a $5 \mathrm{~ms}$ spikerate bin after normalization for the mean spike rate for each channel. The population average was calculated from the animal averages. The recordings used for Figure 8, $A$ and $B$, were performed with wire electrode arrays (500 $\mu \mathrm{m}$ tip separation) as described previously (Hanganu et al., 2006).

Statistical analysis. All population distributions were evaluated for normalicy [Kolmogorov-Smirnov (KS) test] and the appropriate descriptive statistic chosen on this basis, as denoted in the text.

\section{Results}

Spontaneous neuronal activity was recorded from the monocular region of $\mathrm{V} 1$ of head-fixed unanesthetized rats between P5 and P13. Recordings were made during all states of vigilance, although periods of active movement were not routinely analyzed as a caution against movement artifacts. To fully characterize network activity, we used DC extracellular recordings of the local field potential (depth EEG) and multiple unit activity (MUA) (bandpass, 0-5000 Hz) from layer 4 (depth, 300-500 $\mu \mathrm{m}$ from the cortical surface).

In P10 and P11 rats, the most prominent feature of the depth EEG was the presence of steadily recurring large-amplitude negative infra-slow potentials (see Figs. 1, 3A for Wistar examples and $4 D, 5 C$ for Long-Evans examples). These negative potentials had a median duration of $8.75 \mathrm{~s}$ (6-18 s minimum-maximum, $n=80$ events from 8 pups) and a median interval of $60 \mathrm{~s}(41-72$ $s$ minimum-maximum) that were not different between strains. The infra-slow wave was composed of multiple shorter events (Fig. $1 B, C$ ) that resembled spindle bursts described previously during the first postnatal week (Hanganu et al., 2006). The leading negative phase contained 3-15 (median of 5) spindle bursts (duration, $0.5-3 \mathrm{~s}$; median, $0.95 \mathrm{~s}$ ) of field-potential oscillation in the beta band (17-29 Hz peak frequency; median, $21 \mathrm{~Hz}$ ). These bursts of beta-band oscillation were separated by short periods $(0.5-1.5 \mathrm{~s})$ of reduced activity within the larger infra-slow poten- 
A

P10 Visual Cortex
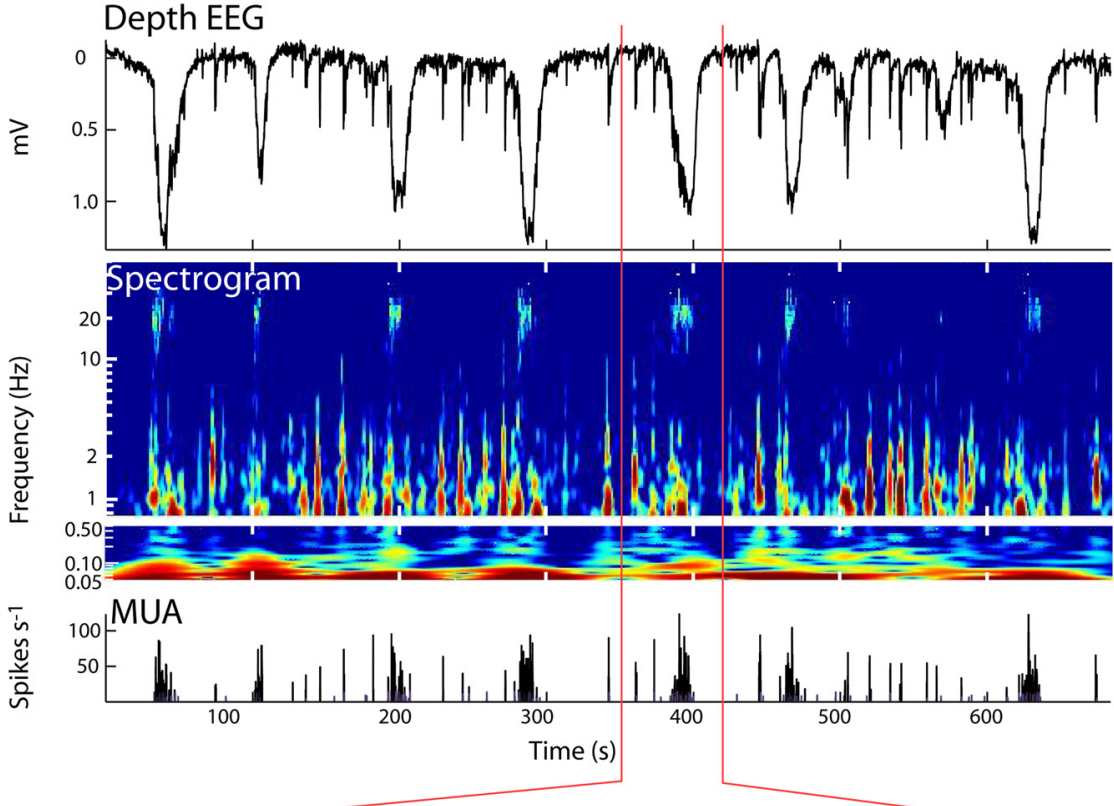

B
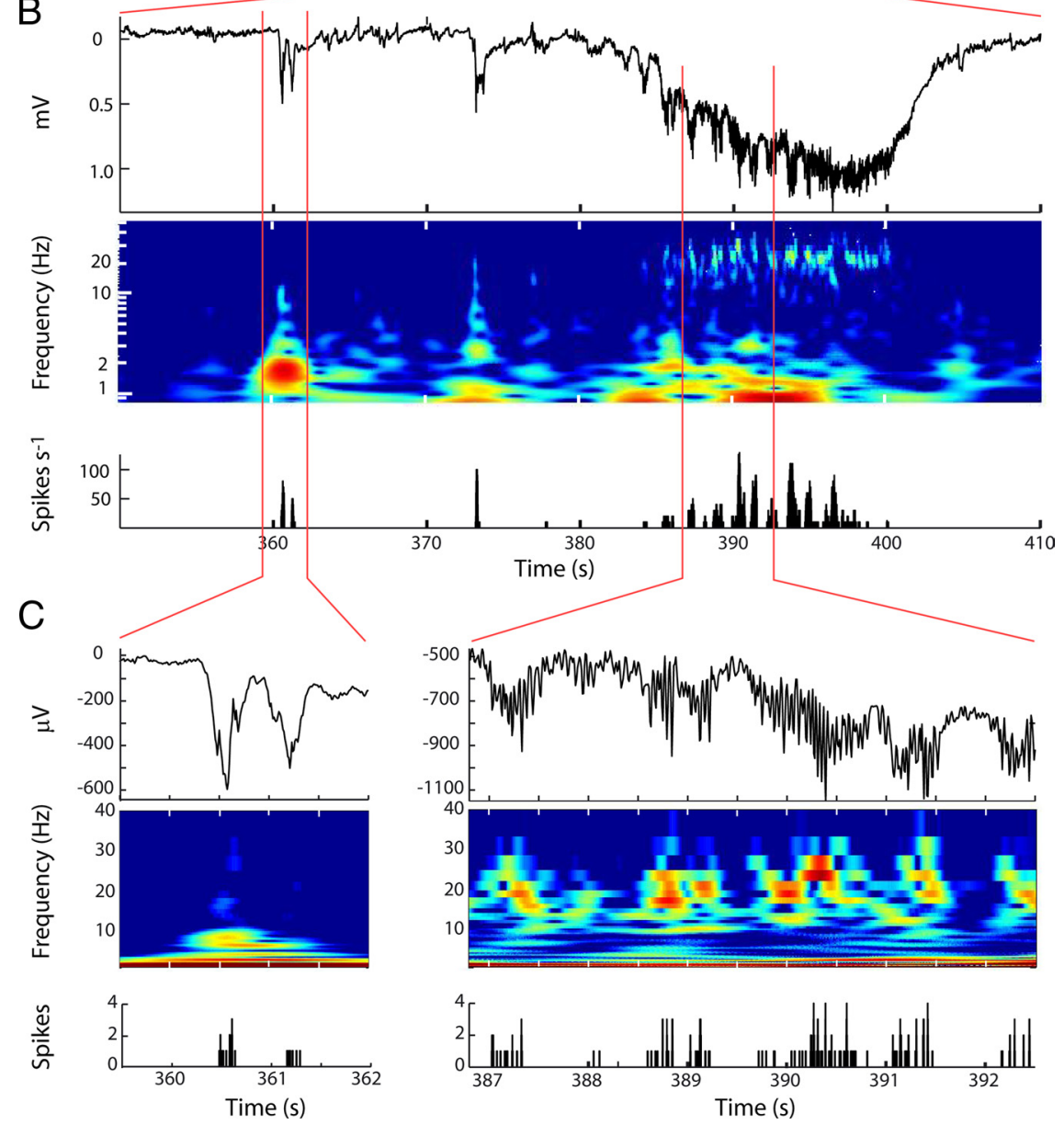

Figure 1. Recurring SATs in visual cortex of infant rats. A, DC depth EEG (layer 4) recordings of spontaneous activity in primary visual cortex of a P10 unanesthetized, head-restrained rat reveal regularly recurring long-duration negative waves. Continuous Morlet wavelet analysis (middle graphs) reveals prominent beta-band oscillations occurring specifically during the infra-slow waves. Frequency spectra in the "standard" $(0.5-50 \mathrm{~Hz})$ and infra-slow $(0.05-0.5 \mathrm{~Hz})$ bands are shown on separate color scales. The presence of infra-slow negative waves containing rapid oscillations is characteristic of human SATs, a name we adopt for this pattern in rats. MUA (bottom graph) is displayed as spike rate in $100 \mathrm{~ms}$ bins. In addition to rat visual SATs, short events (200-2000 ms) of lower amplitude and lacking beta oscillations were also observed. $\boldsymbol{B}$, Expanded time base of the region demarcated in $\boldsymbol{A}$. Higher time resolution shows that SATs consist of multiple bursts of MUA and beta oscillation (spindle bursts) superimposed on the tial. MUA occurred primarily during the spindle bursts and was strongly correlated with the negative troughs of the beta oscillations (see Fig. 7).

As described above, shorter (0.5-3 s) spindle shaped bursts of alpha/beta-band oscillation, named spindle bursts, have been recorded previously during the first postnatal week in V1 and S1. When DC recordings are made, a delta-band component of spindle bursts is observed in S1 (Marcano-Reik and Blumberg, 2008; Minlebaev et al., 2009). However, these negative potentials are much shorter than the infra-slow waves, described here in an immature animal model for the first time. In fact, these infra-slow events resemble multiple spindle bursts occurring in rapid succession. Although spiking events with similar characteristics have been described as "macro-bursts" in ferret cortex (Weliky and Katz, 1999; Chiu and Weliky, 2001, 2002), for the purposes of this paper, we will refer to these events as "rat visual cortex (rv)SATs," or just SATs for short, to emphasize their similarity to the human EEG.

As observed for the first postnatal week (Hanganu et al., 2006), spontaneous activity was characterized by long periods of quiescence between SATs, and isolated action potential activity was rare, with the exception of a population of large units in presumptive layer 5a (see Materials and Methods), which demonstrated persistent tonic activity. Unlike the first postnatal week, however, from P9 we observed spontaneous neuronal activity not associated with spindle bursts/SATs (Fig. 1C). These shorter electrographic events consisted of simple field negative shifts with strong multiunit activity. Unlike SATs and spindle bursts, these short bursts did not display rhythmic oscillations of either the field potential or MUA. We have not analyzed these events in depth in the present study but instead focus on the origins and characteristics of the SATs.

\section{Development of SATs and other cortical activity patterns}

To characterize the development of SATs and other activity, we systematically recorded spontaneous activity between P5 and P13 (1 d before eye opening). Spontaneous activity between P5 and P7 $(n=50$

infra-slow wave. C, Expanded time base for a short burst (left) and an SAT (right) as demarcated in $\boldsymbol{B}$. Short bursts do not have prominent beta-band oscillations. SATs are composed of multiple spindle bursts separated by quiet periods. MUA, shown as the number of spikes in a $5 \mathrm{~ms}$ bin, is associated with the troughs of beta-band oscillations (see Fig. 7). 
events from each of 11 animals) was also marked by the presence of recurring SATs (Fig. $2 \mathrm{~A}$ ). The appearance and duration of the infra-slow component was more variable at these ages, but we observed many SATs with long-duration $(>5 \mathrm{~s})$ negative waves similar to those observed during the second week; however, the beta oscillations and MUA within these waves was shorter (median, $2.2 \mathrm{~s}$ ), resulting in a long return of the field potential to baseline during which there was no significant neuronal activity. The other primary difference between the P5-P7 and P10-P13 animals was the absence of short bursts. Although shorter events (400-1000 ms) were common, these events always had a prominent oscillatory component similar to the longer events, suggesting that they are similar network events of varying length (Fig. 1D).

We examined the developmental trajectory of this apparent dichotomization of activity by quantifying the duration of all observed events between P5 and P13 (at least 200 events from three to four animals at each age). Duration was defined by the continued negative deflection of the DC field potential occurring in combination with an elevated MUA rate. The most apparent change during this period was a widening of the dynamic range for event duration (Fig. $2 \mathrm{~B}$ ). Very long events ( $>5 \mathrm{~s}$ ) were first observed on P8 and continued until the end of the recording period. Short events $(<400 \mathrm{~ms})$ were first commonly observed on P9 and become prominent on P10. Their occurrence continued to increase until P13 by which time they were the large majority of spontaneous events (Fig. 2E). Another dramatic change in the statistics of spontaneous activity was the establishment of a clear separation of short bursts and SATs, because the number of medium length $(2-5 \mathrm{~s})$ events was strongly reduced at approximately P10 (Fig. 2C).

This splitting of events with maturation was also observed in the relationship between event duration and beta oscillations (Fig. 2D). From P5-P7, all events, regardless of duration, displayed a strong rhythmic oscillation with a peak frequency between 8 and $31 \mathrm{~Hz}$ ( $n=50$ events from each of 11 animals). There were two prominent distributions centered at 22 and $10 \mathrm{~Hz}$, although duration was not a determining factor in distinguishing events at each frequency, which likely arose as harmonics of the same oscillation. Thus, in terms of duration and frequency, all events between P5 and P7 appear to be drawn from a single distribution, quantitatively confirming the previous assertion that spindle bursts constitute a singular event during the first week in visual cortex (Hanganu et al., 2006). Between P10 and P11, the two populations of events were clearly separable by both peak frequency and duration, with almost all events $>5 \mathrm{~s}$ displaying a peak frequency between 18 and $30 \mathrm{~Hz}$, whereas shorter events almost always had peak frequencies below $10 \mathrm{~Hz}$. Thus, by the second postnatal week, at least two populations of events can be clearly distinguished.

We further estimated the amount of neuronal activity associated with SATs. Total counts of action potentials over the entire recording session revealed that $87 \pm 5 \%$ (mean $\pm \mathrm{SD} ; n=830$ min recordings $\mathrm{P} 10-\mathrm{P} 11$ ) of layer 4 MUA occurred during SATs.

Beginning at P12 (at least 100 events from eight animals), spontaneous activity became much more continuous and the occurrence of SATs become less regular. We observed long periods of repeating short events that now resembled the slow waves observed during intermediate and deep sleep. At these ages, although SATs still occurred, they were no longer the dominant activity pattern and could often not be separated from ongoing cortical activity. No SATs were observed in the rats older than P14 (30 $\min$ from $n=15$ P14-P19 animals).
The clear segregation of SATs from other activity patterns between P10 and P11 allowed us to study these events in isolation.

\section{Retinal drive of SATs}

We used enucleation of the contralateral eye to test the role of retinal activity in generating SATs. Baseline spontaneous activity was first recorded. Then, as a control for surgery, animals were anesthetized and allowed to recover for $30 \mathrm{~min}$, and baseline spontaneous activity was again recorded. After this, animals were enucleated under the same anesthesia and allowed the same recovery time. Transient anesthesia had no effect on SAT production, but enucleation completely eliminated SATs (Fig. 3). Simultaneous recordings made in V1 ipsilateral to the enucleation showed no change (data not shown). The distribution of event duration was quantified for 50 events from each of the five pups before and after enucleation (K-S test, control vs enucleated, $p<0.0001$ ). The elimination of SATs was accompanied by an increase in events of moderate duration (1-3 s) (Fig. 3B). Interestingly, these moderate length events sometimes displayed prominent rapid oscillations in the field potential and were similar to spindle bursts recorded during the first postnatal week. We examined the effect of enucleation on the generation of rapid oscillations by measuring total frequency power $(1-50 \mathrm{~Hz})$ during $30 \mathrm{~min}$ periods before and after enucleation. Enucleation strongly attenuated the frequencies associated with the rapid component of SATs $(10-40 \mathrm{~Hz}$; peak attenuation at $22 \mathrm{~Hz})$ but increased in power below $10 \mathrm{~Hz}$, reflecting the increased occurrence of short-duration events. Similar results were obtained after suppression of retinal network-driven activity by intraocular injection of urethane (see below). In total, these experiments suggest that, although oscillatory events of short duration can be generated in visual cortex in the absence of sensory input, the generation of long-duration SATs requires retinal input.

\section{Phase III retinal waves have a similar macro-structure as SATs but lack beta oscillations}

We examined spontaneous activity in acutely excised retinas in vitro ( $n=84$ locations from 10 P10-P11 retinas) for evidence of SAT-like activity (Fig. 4). Spontaneous retinal activity in the rats at this age was similar to the patterns described for phase III retinal waves in mice (Kerschensteiner and Wong, 2008; Blankenship et al., 2009). Spiking activity in the ganglion cell layer was grouped into regularly repeating burst clusters (median interval, $53.5 \mathrm{~s}$; median duration, $9.5 \mathrm{~s}$ ) composed of multiple sub-bursts (median, 6). The distributions for the bursts-cluster duration and interburst-cluster intervals revealed extensive overlap and no significant differences from V1 SATs (Mann-Whitney test, $p>$ 0.05 ) (Fig. $4 B$ ). We examined the spike rate autocorrelations of the retinal bursts ( $5 \mathrm{~ms}$ bins; $500 \mathrm{~ms}$ window) for evidence of rapid oscillations similar to those observed during SATs. Unlike V1 SATs, retinal autocorrelation coefficients revealed only a steady decrease with time and no evidence of beta-band oscillations (Fig. 4C).

We tested the hypothesis that the occurrence of SATs is determined by retinal activity by increasing the occurrence of retinal waves via intraocular injection of bicuculline (Bic) and strychnine (Str), which increases the rate of wave initiation in vitro (Blankenship et al., 2009). Contralateral Bic $(20 \mu \mathrm{M})+\operatorname{Str}(40$ $\mu \mathrm{M})$ injection $(5-10 \mu \mathrm{l})$ massively increased cortical activity, resulting in the constant production of alpha-beta oscillations (spindle bursts) separated by short (1-5 s) silent periods. Well defined SATs could not be resolved and were replaced by constantly modulating negative potentials (Fig. $4 D$ ). To quantify the 
A

P6 Spontaneous Activity:Visual Cortex

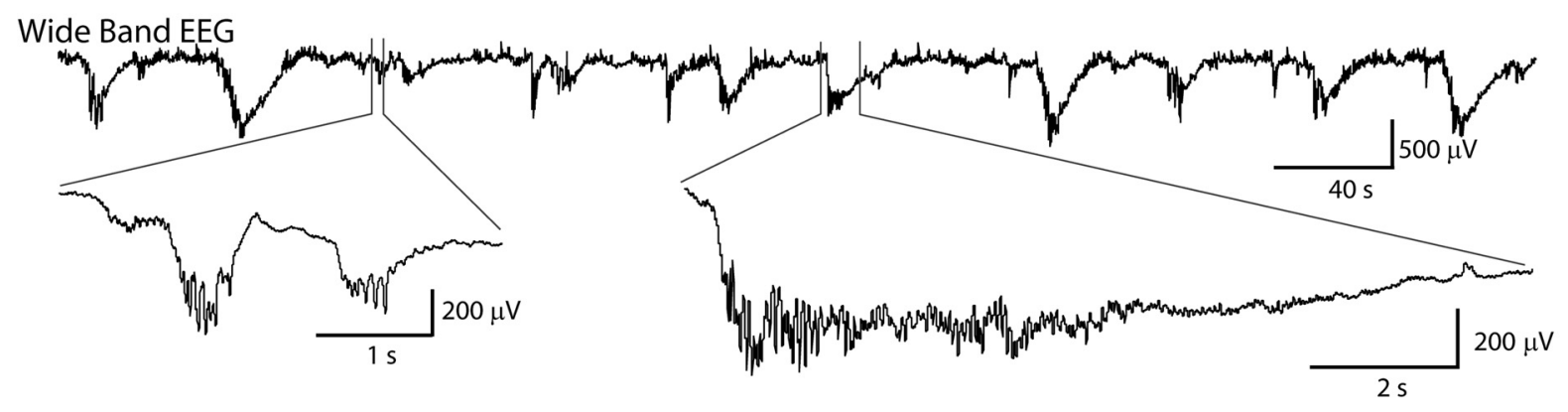

B

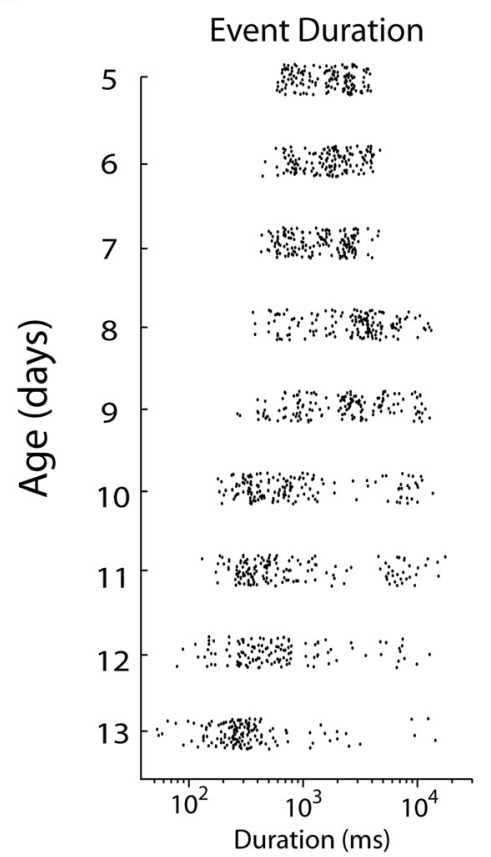

C

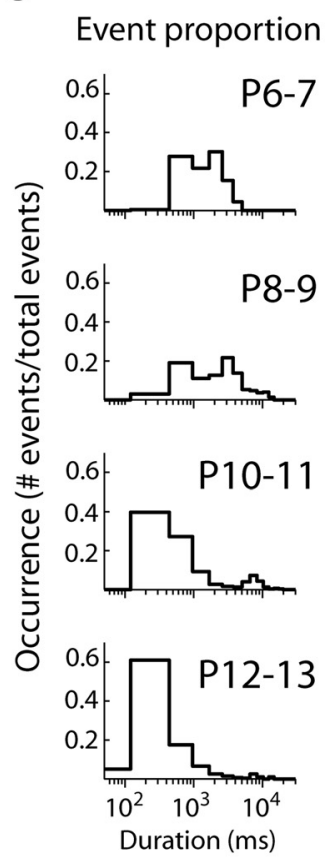

$\mathrm{D}$

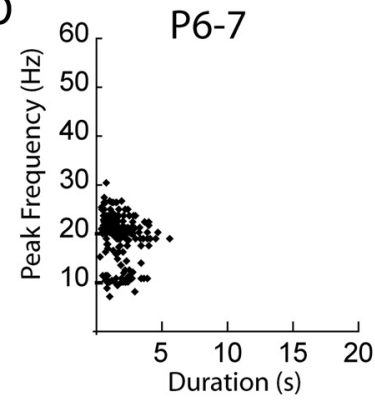

P8-9

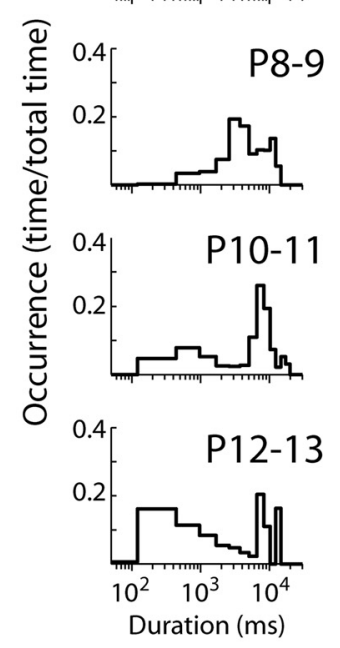

Time proportion
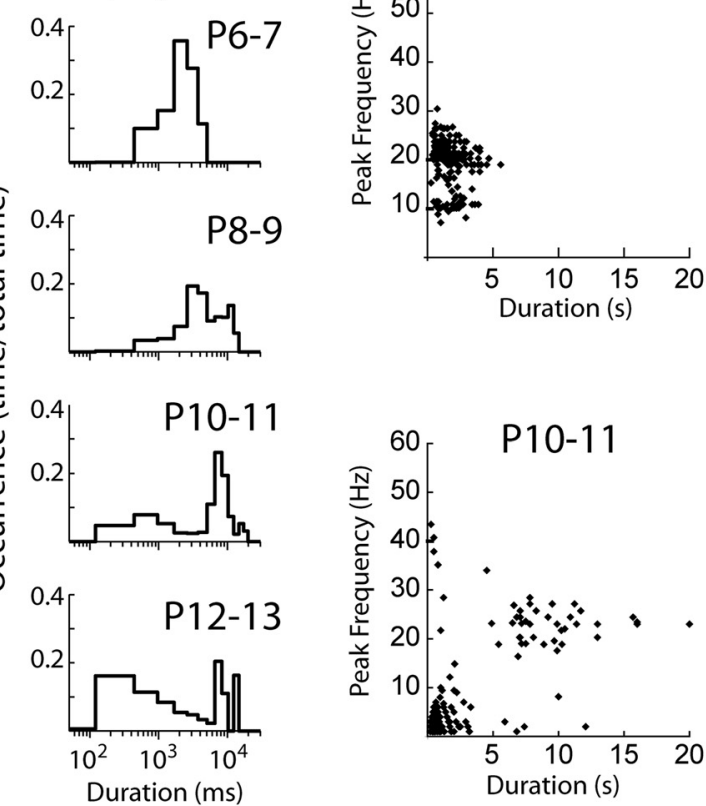

E

P13 Spontaneous Activity:Visual Cortex

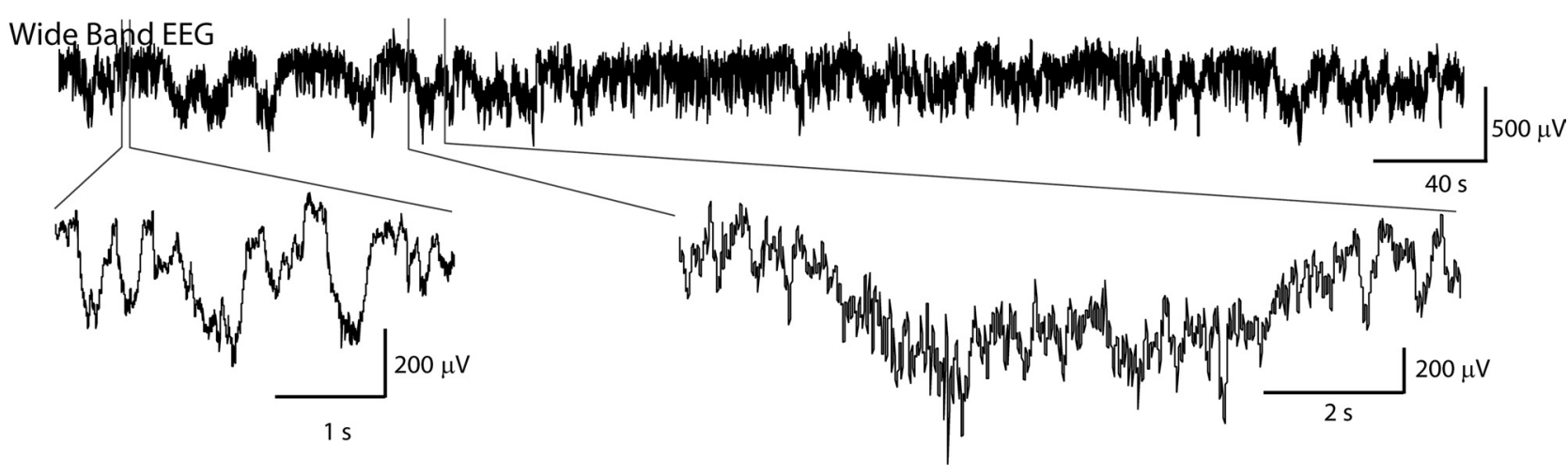

Figure 2. Development of SATs and short bursts. $A$, Wide-band depth EEG recording from layer 4 visual cortex at P6. Infra-slow waves with rapid oscillations were also observed at these ages but with less regularity. Although the potential shift could last 5-10 s, rapid oscillations occurred only during the initial phase of the response (right). Shorter events were also observed, but these also displayed rapid oscillations (left). B, Scatter plot of event duration versus age for 200 events from three to four animals at each age. Maximal event duration increases at P8, and long and short events become clearly separated between P10 and P13. C, Population histograms of all events (50 events each from >6 animals in each age range) showing the log distribution of event duration (left column) or total proportion of time occupied by events of each duration (right). P5-P7 pups show a single continuous distribution of event distribution. By P10 -P11, SATs form a separate distribution from shorter events. Shorter events become more frequent at P12-P13, reducing the proportion of SATs. D, Scatter plots of event duration versus peak frequency $(2-100 \mathrm{~Hz})$ at P6 -P7 and P10 - P11. During the first postnatal week, all events have prominent oscillations above $8 \mathrm{~Hz}$; by P10 -P11, two populations have developed: short low-frequency events and long events with strong oscillations in the beta band. $\boldsymbol{E}$, Wide-band depth EEG from a P13 rat. Activity is essentially continuous at this age, consisting of short bursts and isolated spikes. SATs can still be observed but do not repeat consistently and are often obscured by the ongoing activity. 
effect, we calculated the interval between individual bursts of alpha-beta oscillation (more than three cycles separated by $>500 \mathrm{~ms}$ ) after control injection of ACSF and again after Bic + Str injection. In five of five P10-P11 pups, the distribution of event intervals (50 per animal) was significantly shifted (K-S test, $p<0.001$ in all cases) from a bimodal distribution to an exponential distribution (Fig. $4 E$ ). In four of four animals in which we made simultaneous recordings from ipsilateral V1, no change was observed $(p>0.05)$. The increased activity resulted in a selective increase of frequencies between 20 and 30 $\mathrm{Hz}$ (30 min recordings per pup) (Fig. $4 F$ ).

These results, in combination with the enucleation and intraocular urethane injection experiments, suggest that spontaneous retinal activity is transferred though the lateral geniculate nucleus to cortex to drive SATs. They further predict that the length, sub-burst structuring, and occurrence of V1 SATs (i.e., the macro-structure) are determined by the characteristics of retinal activity, whereas the prominent rapid oscillations that occur during SATs are generated in thalamus or cortex as a result of the burst excitation provided by retinal input.

We examined the forepaw region of S1 for evidence of SAT-like activity (supplemental Fig. 1, available at www.jneurosci.org as supplemental material). DC recordings between P10 and P11 (30 min from $n=3$ pups) showed no sign of the regularly recurring SATs observed in V1. The only infra-slow activity observed was infrequent, irregular, and of very long duration (>30 s). Thus, retinal wave-driven SATs do not appear to propagate to $S 1$, in which activity is instead driven by the topographically appropriate somatic receptors.

\section{SATs are eliminated by low levels} of anesthesia

Because SAT-like mega-bursts were observed in unanesthetized ferret (Chiu and Weliky, 2001) but not anesthetized mouse V1 (Rochefort et al., 2009), we examined the effects of isoflurane and urethane anesthesia on the generation of SATs at P10-P11 (Fig. 5). SATs were eliminated by concentrations of either anesthetic that reduced spontaneous movement but did not prevent foot withdrawal to light squeeze. Under either anesthetic, short bursts remained, and the event distribution was shifted from two clear peaks, to a single peak below 1 s duration $(n=50$ events for each of three pups for each anesthetic, K-S test vs unanesthetized, $p<0.0001$ for both groups). Thus, in V1, SATs are more sensitive to anesthesia than short bursts, and their examination requires the use of unanesthetized animals.

We examined the locus of the anesthetic effect by intraocular injection of urethane (5-10 $\mu 100 \mathrm{~mm})$. Retinal injections rapidly eliminated SATs $(<5 \mathrm{~min})$, but not short bursts, in four of

A P11 Eye Removal
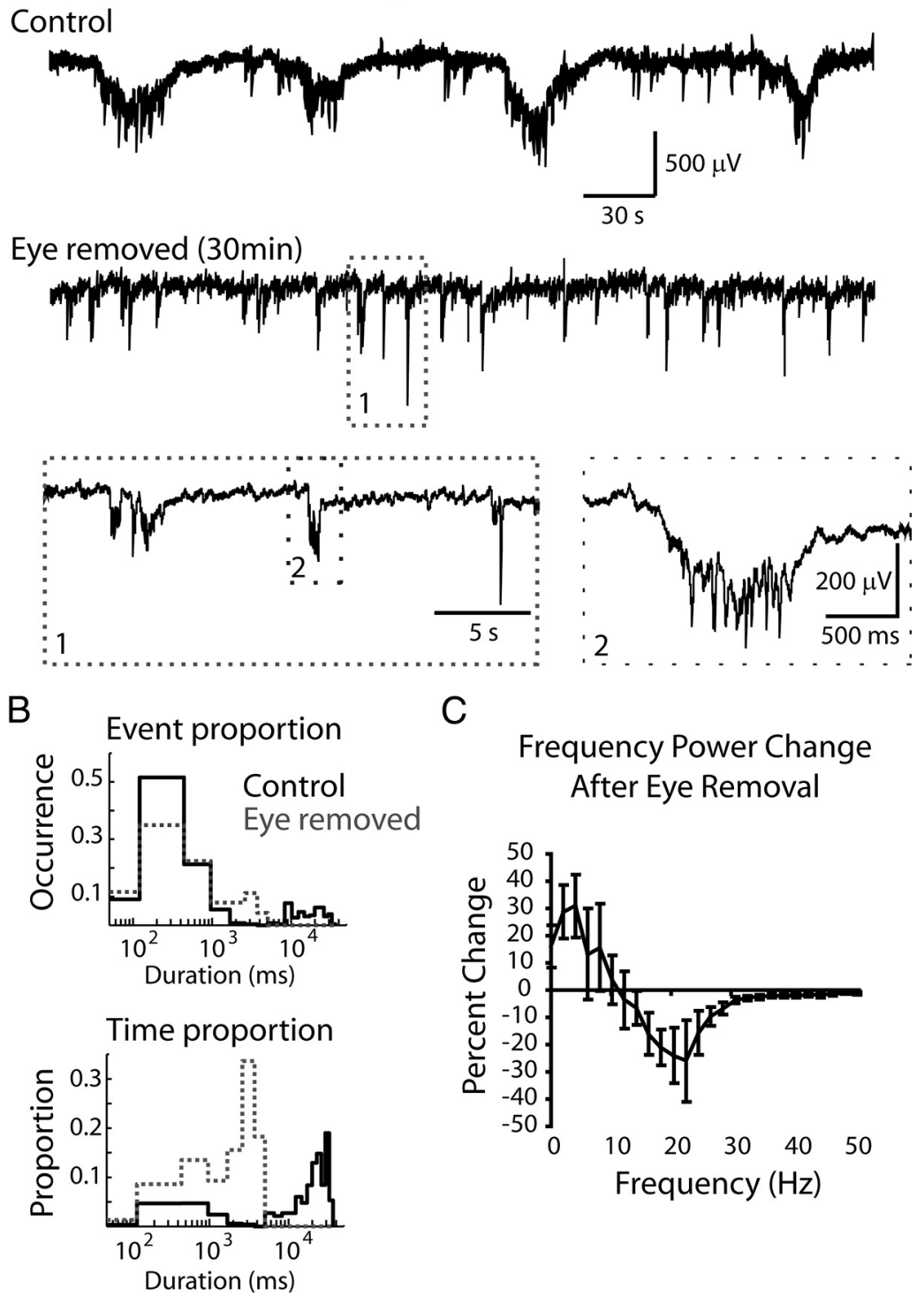

C

\section{Frequency Power Change After Eye Removal}

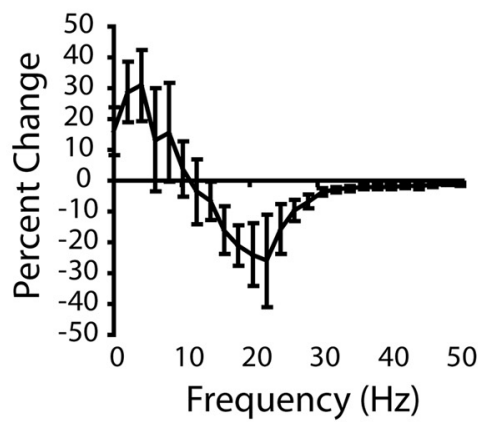

Figure 3. Enucleation eliminates SATs. A, Wide-band V1 depth EEG from a P11 rat before and 30 min after removal of the contralateral eye. After eye removal, short events remain but the repeating SATs are absent. Sequential traces with decreasing time base show the remaining potentials, some of which have more prominent field oscillations than before eye removal. $\boldsymbol{B}$, Histograms of event duration and 5 animals). After eye removal, a new population of medium (2-5s) duration events emerge. C, Percentage change in power spectral density $(1-50 \mathrm{~Hz}$ ) after eye removal ( 30 min recordings from 5 animals; error bars indicate SEM). Eye removal selectively reduces beta-band oscillations associated with SATs but increases power in the lower frequencies as a result of the longer and more frequent short bursts.

four rats (Fig. $5 C)(n=50$ events per animal per condition, $\mathrm{K}-\mathrm{S}$ test ACSF vs injected, $p<0.0001)$. Animals that received retinal urethane injections had more frequent short bursts than intraperitoneally injected rats, suggesting a central locus for shortburst generation but a retinal locus for SATs. These data are complementary to the enucleation experiments because they show that SAT elimination can be accomplished by a less damaging disruption of retinal activity. Furthermore, in total, these data strongly suggest that our short bursts are the same activity recorded by Rochefort et al. (2009) and determined to be a primitive version of "slow-wave" activity.

\section{Synaptic correlates of SATs}

We examined the synaptic composition of SATs with whole-cell voltage-clamp recordings made in close proximity (200-400 
A

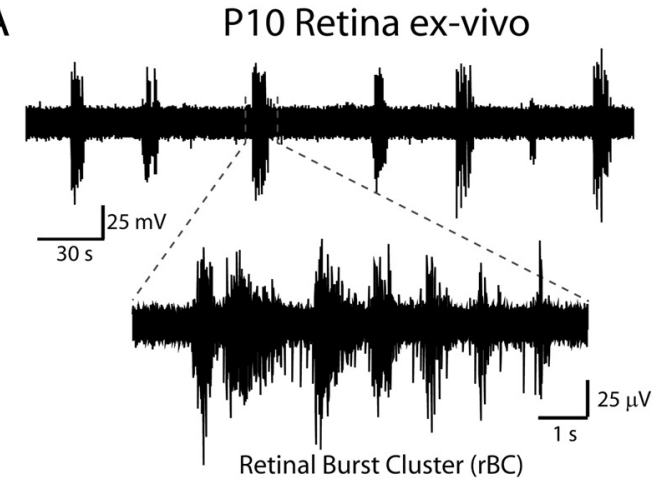

B

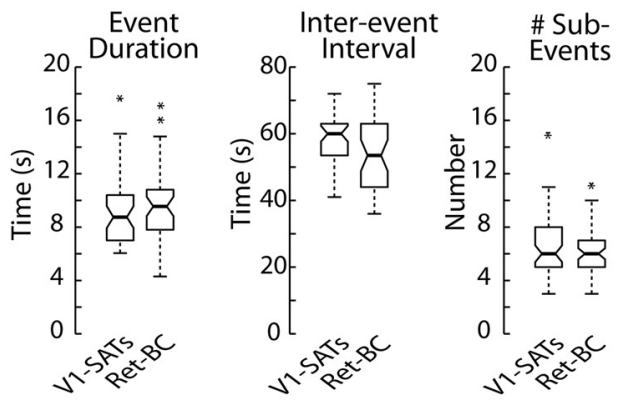

C
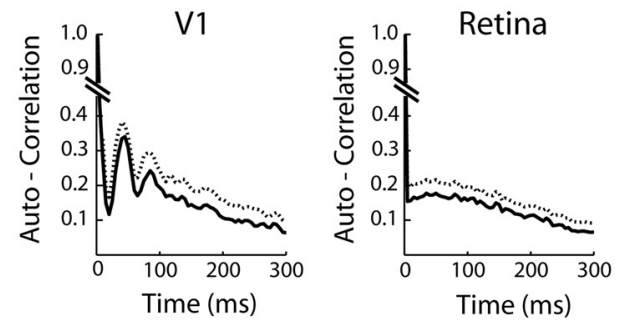

D P10 Depth EEG - V1

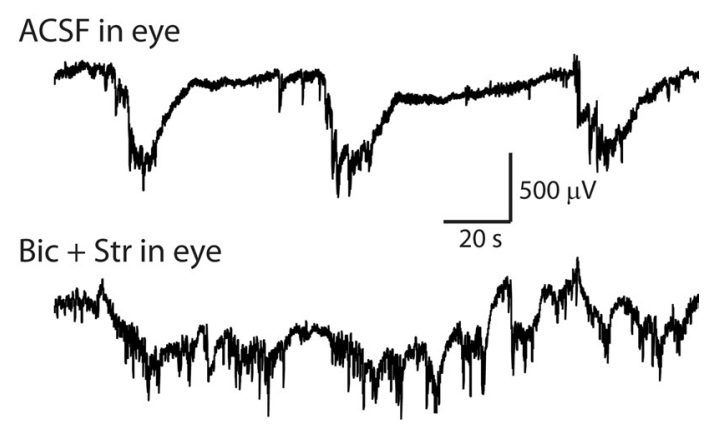

E

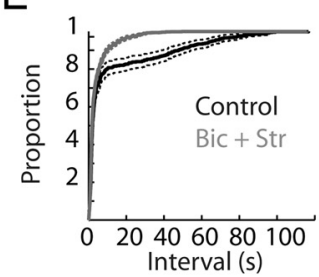

$\mathrm{F}$
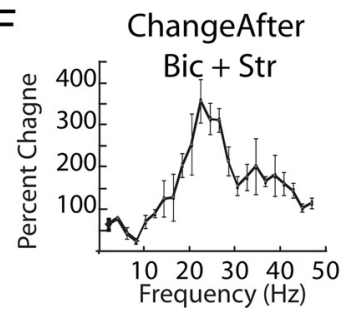

Figure 4. Characteristics of spontaneous retinal waves and visual cortex SATs. A, Extracellular recording of spontaneous activity from an acutely excised retina (P10) shows repetitive, long-duration burst clusters separated by long quiet periods consistent with phase III retinal waves described in mice (Kerschensteiner and Wong, 2008; Blankenship et al., 2009) and similar in structure to SATs observed in visual cortex. $\boldsymbol{B}$, Box plot presentation of duration (left), interevent interval (middle), and number of bursts (right) for V1 SATs and retinal burst clusters ( $n=10$ SATs from each of $8 \mathrm{P} 10-\mathrm{P} 11$ pups, 10 burst clusters from each of $10 \mathrm{P} 10-\mathrm{P} 11$ retinas). Middle line shows median, the box ends at the 25th and 75th percentiles, and the whiskers show the total range. Outliers ( $>1.5$ times the intraquartile distance) are plotted with asterisks. $\mu \mathrm{m}$ ) to the field electrode (Fig. 6). Neurons located 300-600 $\mu \mathrm{m}$ below the cortical surface ( $n=5$ from 3 animals) showed a close correspondence between negative field-potential deflections and whole-cell glutamatergic synaptic currents $(-80 \mathrm{mV}$ holding potential). Both the infra-slow and rapid components of the SAT could be observed in the single-cell currents. The infra-slow potential was associated with a similar slow current flow, whereas the rapid oscillations were closely locked with fast synaptic currents. Cross-correlation of whole-cell currents and the rapid field oscillation (both signals high-pass filtered above $2 \mathrm{~Hz}$ ) showed a strong peak correlation (mean $\pm \mathrm{SD}$ correlation coefficient of $0.4 \pm 0.1$, five SATs per animal). Cross-correlation during the inter-SATs times showed an equally strong correlation but spread over a wider time base. These results together with previous findings in barrel cortex suggest that the rapid oscillations of SATs are generated by rhythmic AMPA receptor-mediated synaptic currents at beta-frequency range, whereas the infra-slow potential is likely generated by summation of NMDA, AMPA, and kainate receptor-mediated currents (Minlebaev et al., 2009).

\section{Beta oscillations of SATs synchronize activity in superficial layers}

The depth profile of SAT-related activity was examined with multisite linear electrode arrays (Michigan probes, $100 \mu \mathrm{m}$ spacing) (Fig. 7A). These recordings showed that MUA was elevated in all cortical layers during SATs, and this firing, as in layer 4, was organized into multiple sub-bursts (Fig. $7 B$ ). One exception to this was presumptive layer $5 \mathrm{a}(500-600 \mu \mathrm{m}$ depth), which often contained units that exhibited tonic firing between bursts as well as between SATs. The beta-band field-potential oscillations, in contrast, were localized to superficial layers (layers 2-4), with a peak 300-400 $\mu \mathrm{m}$ below the pial surface (Fig. 7C,D). Close examination of the temporal relationship between these beta oscillations and MUA revealed additional differences between layers. In superficial layers, MUA occurred primarily during the troughs of the beta oscillation and less frequently during bursts of activity with smaller field deflections. Unit firing in deeper layers, however, was not strongly coordinated by the beta oscillations, and we did not observe another pattern that organized firing in the deep layers. A depth profile of the beta oscillations was constructed by phase averaging centered on the negative peak of the layer 4 trough (Fig. 7D) (1000 events from each of eight pups). CSDs calculated from these triggered averages show a prominent sink in layer 4 that ascended to superficial layers. Average spike

\section{$\leftarrow$}

Notches show the comparison interval (95\% confidence). For each parameter, the distributions of retinal waves and V1 SATs overlap and are not significantly different (Mann-Whitney U test, $p>0.05$ ), suggesting that the occurrence, duration, and burst structure of cortical SATs are determined by retinal activity. $C$, Autocorrelation of MUA spike rate ( $5 \mathrm{~ms}$ bins) from layer $4 \mathrm{~V} 1$ and retina, same population as $\boldsymbol{B}$. Dark line indicates mean, and dotted line indicates SD. V1 recordings display evidence of structure in the autocorrelation as a result of the beta-band oscillations, whereas retinal recordings display only a simple drop off with time. This suggests a nonretinal (i.e., thalamic or cortical) generator for the rapid oscillations. $\boldsymbol{D}$, Increasing retinal wave initiation via intraocular injection of Bic and Str (Blankenship et al., 2009) increases occurrence of visual cortex SATs. After control ACSF injection, repetitive SATs are observed as in non-injected rats (top trace). Bic + Str injection (10 min) caused a massive increase in the occurrence of SATs (bottom trace). $\boldsymbol{E}$, Interevent interval distribution after ASCF and Bic + Str injection. Control cortical activity (black lines) showed a bimodal distribution with a large shoulder at 20-60 s. After injection (gray lines), the interval shifted to an exponential distribution ( $n=50$ events from each of 5 P10-P11 pups). Dotted lines indicates $95 \%$ confidence interval. $\boldsymbol{F}$, Percentage change in power spectral density $(1-50 \mathrm{~Hz}$ ) after intraocular injection (30 min recordings from 5 animals; error bars indicate SEM). Increasing retinal wave initiation selectively increased beta-band oscillations. 
rate ( $5 \mathrm{~ms}$ bins) in layers $2-4$ was highest in the trough and suppressed between phases. Mean spike rate in the deeper layers was much lower than in superficial layers but showed a small increase after the layer 4 trough.

Because of differences in sampling and baseline spike rate between layers, we further examined the synchronization of activity by beta oscillations by calculating correlation coefficients for normalized spike rates ( $5 \mathrm{~ms}$ bins) between layers (Fig. $7 E)$ (10-15 SATs from each of eight pups; correlation coefficients were generated for each animal, and the reported mean is the average of eight pups). MUA rate changes recorded at electrodes placed in layers $2 / 3$ and 4 were strongly correlated with each other (mean \pm SD correlation coefficient, $0.52 \pm 0.13$ for adjacent electrodes), but only weakly correlated to sites located in deep layers (mean \pm SD correlation coefficient, $0.21 \pm 0.14 \mathrm{SD}$; $t$ test, $p<0.0001$ ). Even adjacent electrodes in layers 5/6 were less strongly correlated than similarly spaced electrodes in the superficial layers (mean $\pm \mathrm{SD}$ correlation coefficient, $0.30 \pm 0.08 \mathrm{SD} ; p<0.0001)$. The temporal relationship between the rapid $(>2$ $\mathrm{Hz}$ ) component of the field potential and MUA in each layer were further quantified by cross-correlation analysis (Fig. $7 F)$. As expected from the trough averaging, spike rates in superficial layers were positively correlated at $0 \mathrm{~ms}$ lag with spike-rate modulations on adjacent electrodes and negatively correlated to the field potential in superficial layers. Peak correlation coefficients for layer 6 occurred with slight delay (mean $\pm \mathrm{SD}$, $5.4 \pm 2.5 \mathrm{~s}$ ) relative to layer 4 , a relationship that was also apparent in the negative correlation to field potentials at the same delay.

In total, our data show that the beta oscillations specifically synchronize activity within a cortical column consistent with data from the somatosensory cortex (Dupont et al., 2006; Yang et al., 2009). This effect is strongest for superficial layers and more modest in deep layers.

\section{Beta oscillations synchronize superficial layers as a spreading wave}

Phase III retinal waves comodulate firing at locations separated by hundreds of micrometers (Blankenship et al., 2009). We examined how this spread correlates activity patterns within and between cortical hemispheres by recording with horizontal wire electrode arrays placed in both cortices (Fig. 8A). Electrodes within the same hemisphere (500 and $1000 \mu \mathrm{m}$ separation, $n=$ 30 SATs from each of 10 P10-P11 animals) were likely to record SATs with high temporal proximity. On average, $59 \pm 8 \%$ (mean \pm SD) of SAT starts on one electrode were within $2 \mathrm{~s}$ of the start of a SAT on electrodes on the same side, and $86 \pm 5 \%$ occurred within 5 s. In total, 79\% of SATs occurring on one electrode overlapped by at least $1 \mathrm{~s}$ SATs at $500 \mu \mathrm{m}$ distance.
SATs separated by $>10$ s were very rare, consistent with the clustering of retinal waves observed in vitro by us and others (Kerschensteiner and Wong, 2008; Blankenship et al., 2009). In contrast, we observed no correlation in SAT occurrence between hemispheres, consistent with the retinal generation of SATs, poor ipsilateral representation at this age (Smith and Trachtenberg, 2007), and impaired decussations of albino rats (Lund, 1965).

The horizontal synchronization of cortical activity during SATs was examined with four shank multielectrode arrays placed along the rostrocaudal axis $(4 \times 4200 \mu \mathrm{m}$ separations) (Fig. 8C). Recordings of 20 SATs from each of four pups each showed the same pattern: SATs often occurred simultaneously on multiple shanks but were also observed to spread between them, consistent with the spread of activity as a random wave front. Slow spreading activity ( $>100 \mathrm{~ms}$ per $1 \mathrm{~mm}$ ) was always observed in the horizontal (between shanks) and not vertical (between layers) direction. When SATs were recorded simultaneously on multiple shanks, the beta oscillations in layer 4 were always synchronous (Fig. $8 D, E$ ). Sites that became engaged by the spreading SAT developed beta oscillations synchronized to the other electrodes; we never observed nonsynchronous oscillations during SATs in the same hemisphere. In contrast, SATs in opposite hemispheres were never synchronous (data not shown; $n=30$ SATs from each 
A

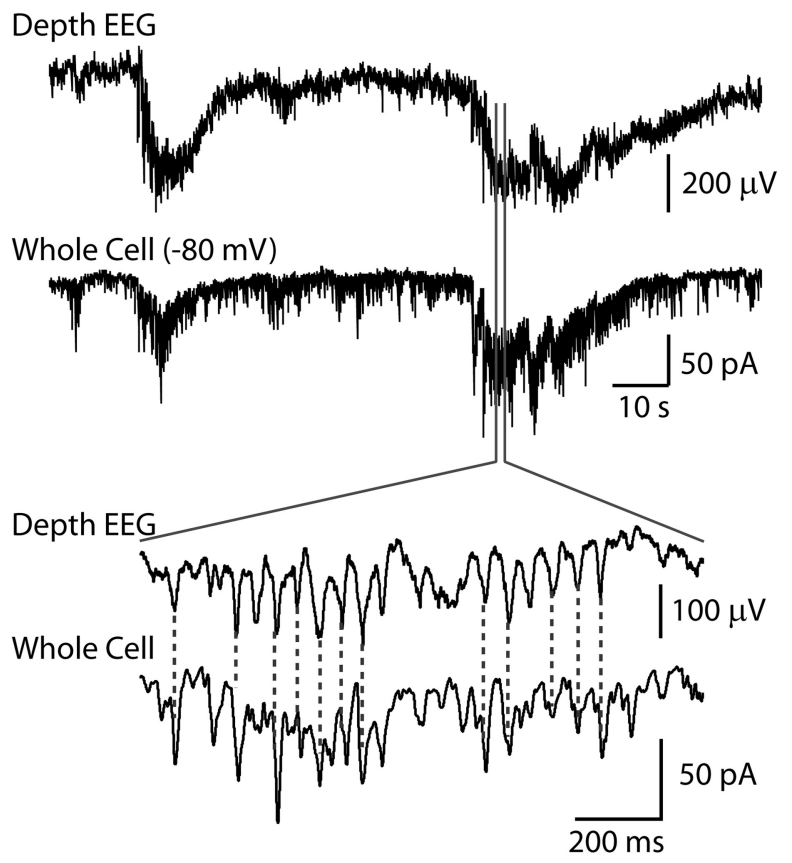

B Whole Cell vs. Depth EEG

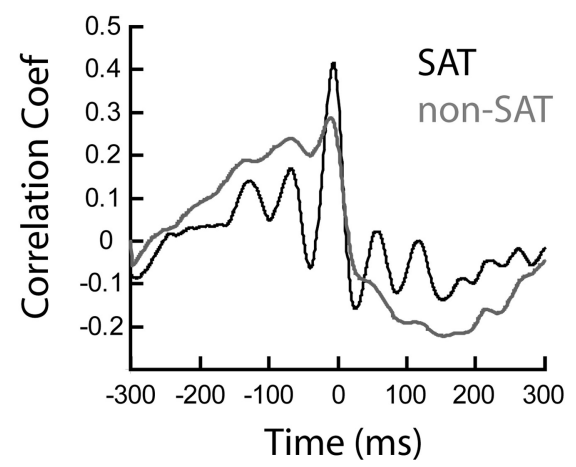

Figure 6. Synaptic correlates of SATs. $A$, Simultaneous whole-cell ( $400 \mu \mathrm{m}$ depth, $-80 \mathrm{mV}$ holding potential) and nearby depth EEG (field-potential) recordings show that the infra-slow wave of SATs is composed of a similar long-duration depolarizing current. Bottom, An expanded time base shows that rapid oscillations in the field potential are closely correlated with synaptic whole-cell currents. $\boldsymbol{B}$, Cross-correlation of fast ( $>2 \mathrm{~Hz}$ ) whole-cell currents and depth EEG shows synchrony of beta oscillations and synaptic currents (black line) from one cell. A similar cross-correlation during inter-SAT times shows that this high-frequency correlation is unique to SATs (gray line). A similar relationship was observed in five of five neurons recorded from three pups.

of 10 P10-P11 animals). The relative role of this synchrony in coordinating activity laterally and vertically was measured by computing the correlation coefficient between normalized spike rates in layer 4 and layers 5/6 during SATs. In all four pups, electrodes on adjacent shanks in layer 4 were more highly correlated than those in the deeper layers on the same or adjacent shafts (Fig. 8F). Population averages showed that, for a given separation, layer 4 electrodes were more correlated to other layer 4 electrodes than to deeper electrodes (Fig. 8G). The average \pm SD correlation coefficient of layer 4 electrodes separated by 200 $\mu \mathrm{m}$ was $0.68 \pm 0.04$ but $0.36 \pm 0.02$ ( $t$ test, $p<0.0001$ ) to the electrode on the same shaft $200 \mu \mathrm{m}$ deeper. A similar relationship held for shafts separated by 400 and $600 \mu \mathrm{m}$. In contrast, electrodes located in layers 5 or 6 had correlation coefficients of $0.38 \pm 0.06($ mean $\pm \mathrm{SD})$ for electrodes with $200 \mu \mathrm{m}$ horizontal separation and $0.39 \pm 0.02$ for the same vertical distance ( $t$ test, $p=0.93)$.

In total, the data on horizontal spread are consistent with the hypothesis that SATs are driven by retinal waves and that they synchronize synaptic and spiking activity in superficial layers via the beta oscillations.

\section{Discussion}

The main findings of the present study are as follows: (1) spontaneous activity in the rat visual cortex during the second postnatal week is organized in regularly occurring long-lasting episodes of highly rhythmic beta oscillations nested in a largeamplitude infra-slow negative potential, which share many characteristics with the SATs described in preterm human EEG; (2) rvSATs are driven by phase III spontaneous retinal waves and patterned by the thalamocortical circuit to produce beta oscillations that synchronize activity in the superficial layers at coactive locales. Thus, rvSATs appear to be the cortical response to input from the sense organ, not an internally generated spontaneous cortical activity. Finally, our data show that rvSATs are clearly separate from ongoing cortical activity, which had a separate developmental profile.

\section{SATs in rodent, human, and ferret}

Several lines of evidence indicate that rvSATs described in the present study are homologous to preterm SATs. In both species, SATs are regularly occurring events of comparable duration, associated with large infra-slow potentials and rapid oscillatory activity (Tolonen et al., 2007). Human SATs are expressed in sensory cortices, predominantly in occipital areas (Vanhatalo et al., 2005). SATs in both species occur during comparable stages and share a common developmental profile, becoming longer by accumulating multiple short events before finally disappearing around the onset of vision (birth in humans and eye opening rats). They develop in counterpoint to the diversification and increasing continuity of ongoing cortical activity and the acquisition of coherent sleep states [second postnatal week in rats and gestational week 36-40 in humans (Jouvet-Mounier et al., 1970; Lamblin et al., 1999; Blumberg et al., 2005)]. Despite the remarkable similarity, differences also exist. First, SATs in human often occur synchronously in both hemispheres, whereas in the rat, SATs are uncorrelated between hemispheres. The binocularity of human vision predicts that retinal activity in both eyes should drive activity in each cortex, as observed in ferrets (Chiu and Weliky, 2002). Such a mechanism can partially explain the higher SAT rate in human $(\sim 0.1 \mathrm{~Hz})$ compared with rat $(\sim 0.02 \mathrm{~Hz})$, although species differences in spontaneous retinal activity will contribute in currently unknown ways (Warland et al., 2006). Finally, whereas rvSATs are dominated by a highly regular beta oscillation, human SATs nest activity in a wider range of frequencies. This is likely attributable to the poor transmission of the beta oscillations to the surface (Fig. $7 C, \mathrm{Mz}$ ), as well as the complex propagation and summation of dipoles from multiple columns to the large surface electrodes in human. Ferret mega-bursts are similar in length, sub-burst structure, and retinal dependency as rodent SATs (Chiu and Weliky, 2001); they also demonstrated evidence of $10 \mathrm{~Hz}$ rapid oscillations (the lower frequency consistent with their recording location in layer 5/6).

In summary, we propose that, in occipital cortex, SATs in rats and humans and mega-bursts in ferrets constitute a homologous class of events whose differences arise as a result of differences in binocularity, spontaneous retinal activity, and recording techniques. We further suggest that these activities are not function- 


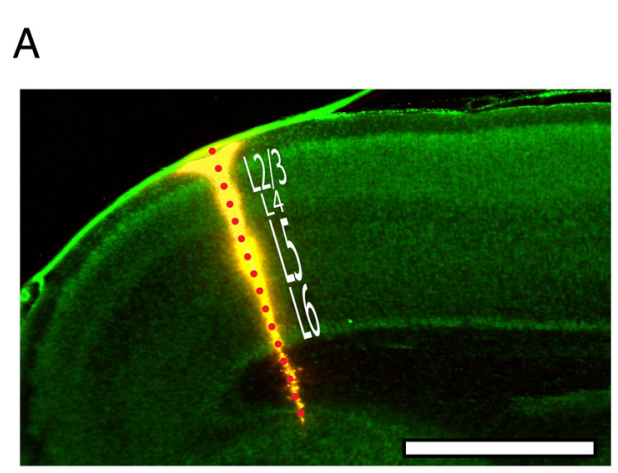

B

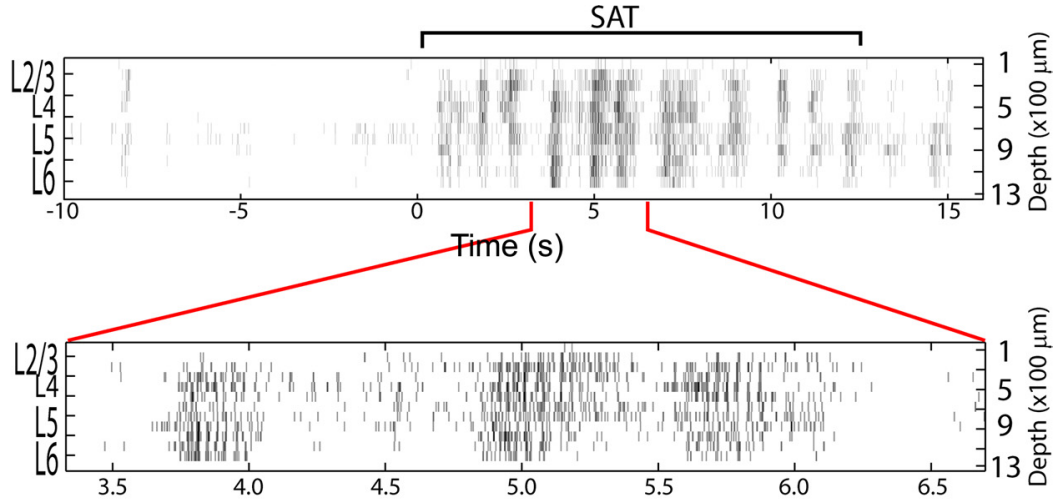

C

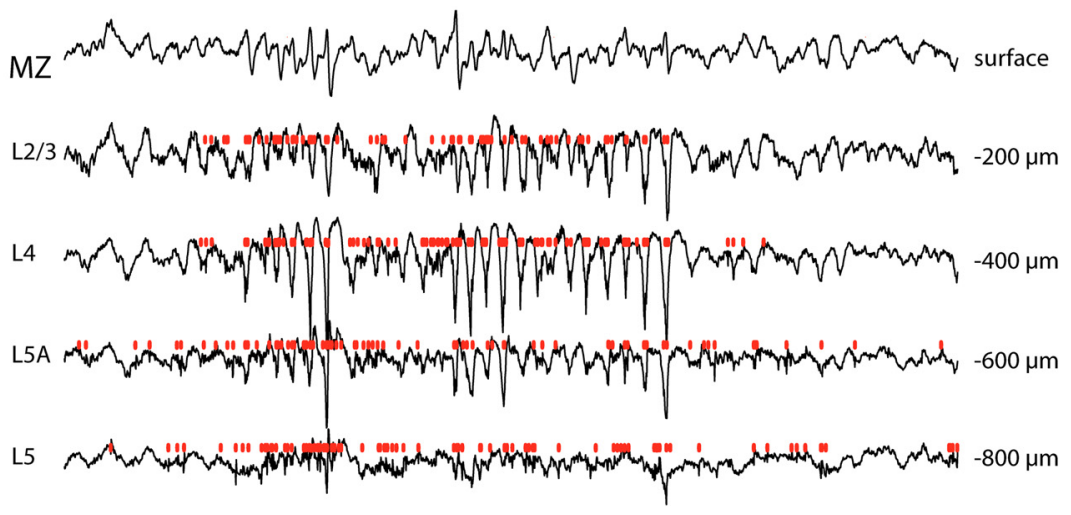

$\mathrm{D}$

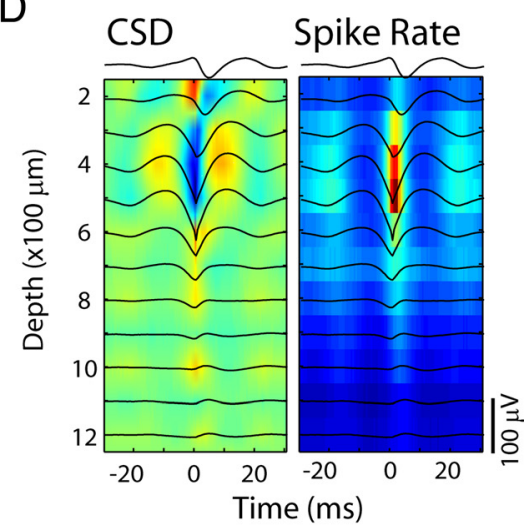

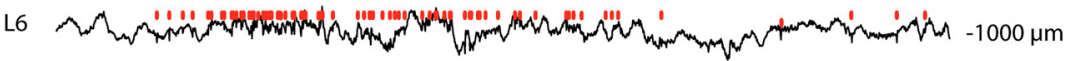

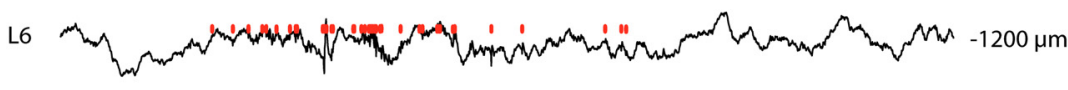

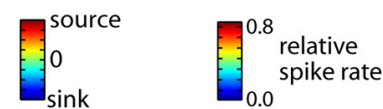

$200 \mu \mathrm{v}$

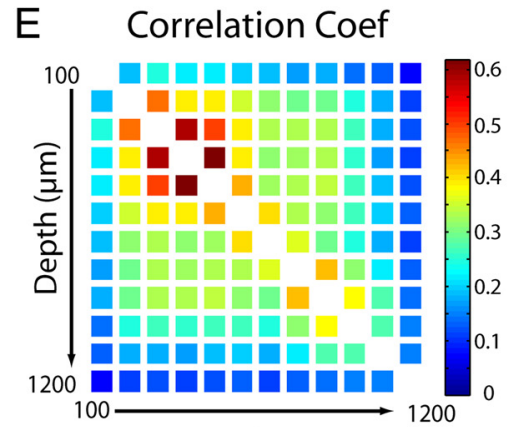

Depth $(\mu \mathrm{m})$
$\mathrm{F}$

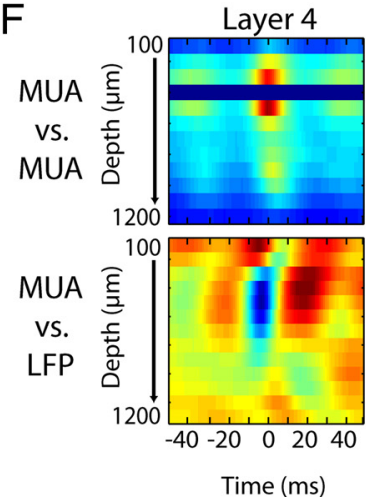

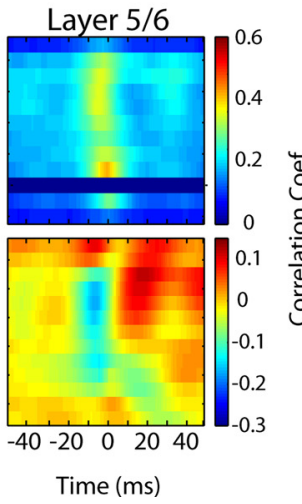

Figure 7. Beta oscillations during SATs synchronize activity in superficial layers. A, Depth recordings using 16-site linear electrode arrays (100 $\mu \mathrm{m}$ contact separation) were used to characterize laminar activity during SATs. Photomontage of a postmortem sagittal slice (florescent Niss I stain in green) with labeled probe tract (yellow). Approximate locations of the recording sites are marked by red dots. Scale bar, $1 \mathrm{~mm}$. B, Spike rasters through the depth of cortex show multiple bursts of activity in all layers during SATs and lack of firing during the inter-SAT period. The approximate start of the SAT is time $0 \mathrm{~s}$. Bottom, Three sub-bursts are plotted on an expanded time base to show the activation of all layers during and between sub-busts. $C$, Field potential $(>2 \mathrm{~Hz})$ and spikes (red dots) for alternating electrodes during a single sub-burst. Beta-band field oscillations are largest in layer 4 and restricted to superficial layers in which they synchronize MUA. Activity in deeper layers, although higher during the burst, is not tightly correlated to the field oscillations. B, C, P10 animal. D, CSD and MUA rate of beta-band oscillations calculated using the peak of the layer 4 trough triggered averages $(5 \mathrm{~ms}$ bins, $n=8$ pups; $P 10-P 11,>1000$ troughs per pup). Average field potential is overlaid. Rapid oscillation sinks originate in layer 4 and ascend to superficial layers. This sink is associated with high spike rates in layers 4 and $2 / 3$. E, Average correlation coefficient of spike rates ( $5 \mathrm{~ms}$ bins) across all electrodes during SATs (10 SATs from each of 8 pups). Layers 2/3 and 4 are highly correlated, with much lower correlations among other cortical layers. $\boldsymbol{F}$, Average cross-correlation matrix between spike rates in layer 4 (left) or layer $5 / 6$ (right) versus spike rate (top) and field potential (bottom). Layer 4 cross-correlation confirms the field-potential averages (D) showing that spike rates have a high cross-correlation coefficient with zero lag in superficial layers and a negative correlation with the field potential. Layer $5 / 6$ cross-correlation shows a positive correlation with slight delay to layer 4 spiking and fields; however, the absolute magnitude of correlation is lower, suggesting a more independent spike-firing mechanism.

ally distinct from spindle bursts described previously in the rat pups during the first postnatal week in V1 (Hanganu et al., 2006) and delta brushes observed in premature human and rat somatosensory cortex (Khazipov et al., 2004; Milh et al., 2007; Minlebaev et al., 2009) but are long-duration versions of these patterns that arise because of the developmental increase in duration of the retinal waves. rvSATs, in fact, appear to be multiple spindle bursts occurring sequentially, which has also been suggested as a 
A

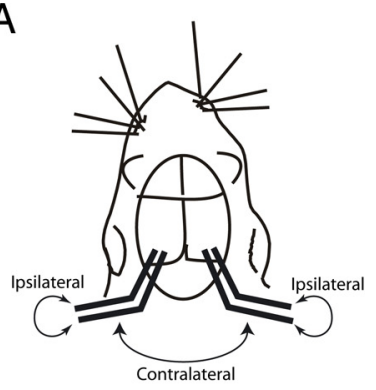

B

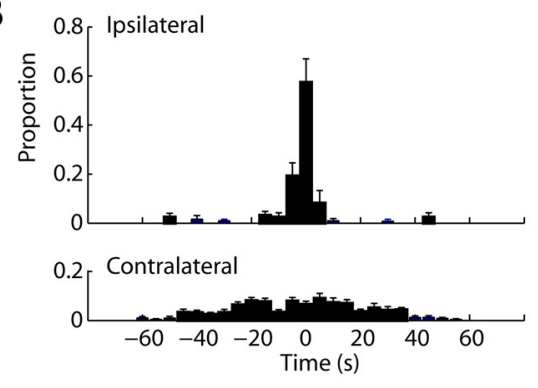

D
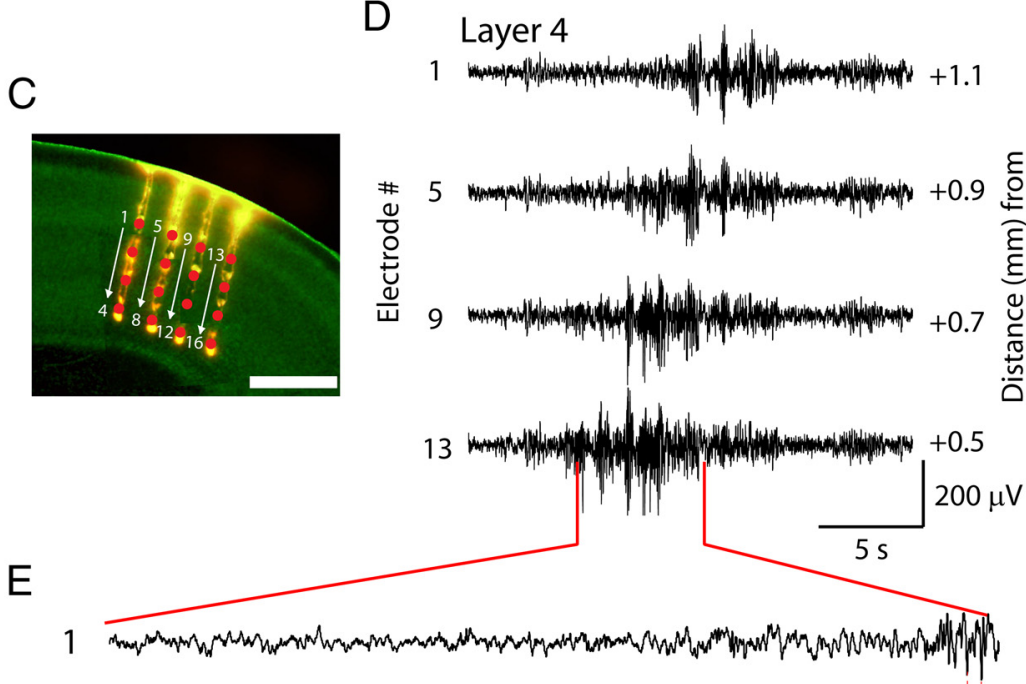

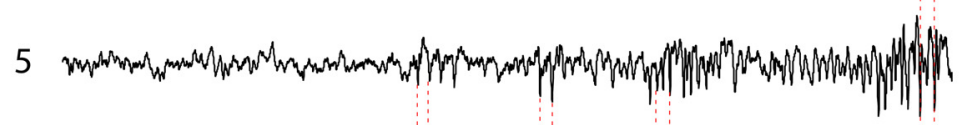

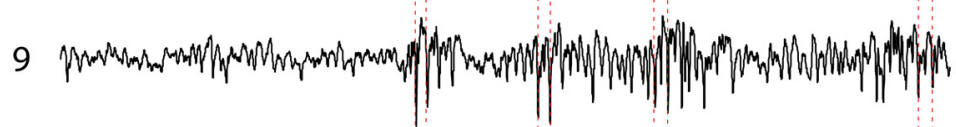

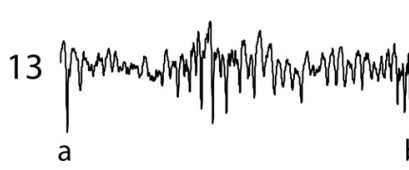

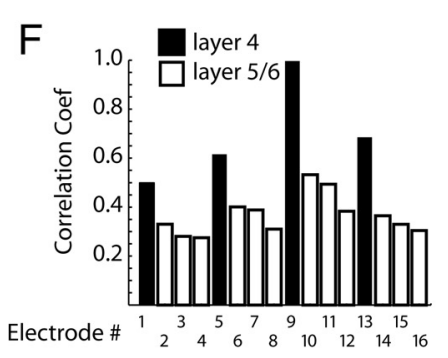

G
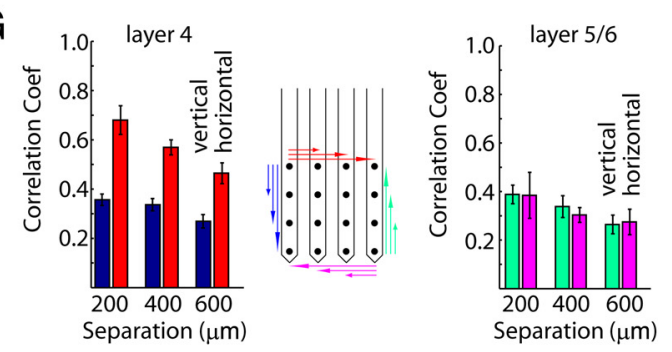

Shaft \#

Figure 8. SATs synchronize superficial layers as a spreading oscillation. $\boldsymbol{A}, \boldsymbol{B}$, Wire electrode arrays recording activity simultaneously in both visual cortices shows that the occurrence of SATs is correlated within ipsilateral, but not contralateral, cortex. Calculation of the closest SAT initiation time between same-side (top; 500-1000 $\mu \mathrm{m}$ separation) and opposite-side (bottom) electrodes. For same-side electrodes, $>60 \%$ of SATs occur within $2 \mathrm{~s}$ of each other, whereas there is no correlation between opposite-side electrodes, suggesting independent generation of contralateral SATs but common-mode generation of ipsilateral SATs. C, Depth recordings using a 16-site, four-shank ( $4 \times 4200 \mu \mathrm{m}$ separation) electrode array was used to characterize the horizontal and vertical spread of SATs. Photomontage of a postmortem sagittal slice (florescent Nissl stain in green) with labeled probe tracts (yellow). Approximate locations of the recording sites are marked by red dots along with the electrode number referenced in D-F. D, Example SAT observed traveling across all four layer 4 electrodes in a P10 rat. $\boldsymbol{E}$, Expanded time base showing that, as SATs spread, the beta oscillations synchronize activity in adjacent layer 4 sites (dotted lines). $\boldsymbol{F}$, Spike-rate (5 ms bins) cross-correlation between all electrodes in a single animal (20SATs). The correlation coefficient relative to electrode 9 (layer 4) mechanism for increasing SAT length in humans (Vanhatalo and Kaila, 2006). Consistent with the hypothesis that the duration and macro-patterning of SATlike activity (infra-slow waves and alphabeta oscillations) is determined by cortical input, long-duration alpha-beta oscillations observed in rat barrel cortex (Yang et al., 2009) (supplemental Fig S1, available at www.jneurosci.org as supplemental material), have different duration and incidence than V1 SATs, and require repetitive stimulations to evoke them, suggesting a different driver (somatic vs retinal).

\section{Network mechanisms of SATs}

The following network model of rvSATs best fits our data: spontaneous retinal burst clusters provide excitatory input to the thalamocortical circuitry (Mooney et al., 1996), which then oscillates at beta frequency. Each retinal burst likely drives a component spindle burst whose currents summate to form the infra-slow wave. If the beta oscillations arise in thalamus, cortex, or the thalamocortical loop is not clear. The CSD profile of beta oscillations is consistent with a thalamic origin (Molnár et al., 2003), but the ability of isolated cortex to produce similar oscillations (Dupont et al., 2006) suggests that intrinsic cortical dynamics also contributes. The horizontal spread of rvSATs in visual cortex is also consistent with their generation by retinal waves. Thus, our results suggest that V1 SAT activity is directly driven by peripheral input, but it remains possible that individual spindle bursts within the SAT are triggered by retinal bursts. During the first postnatal week, brief electrical inputs have been shown to trigger short-duration spindle bursts (Hanganu et al., 2006; Minlebaev et al., 2007; Yang et al., 2009). Thus, beta oscillations may have two modes of generation: short SATs (500 ms to $1 \mathrm{~s}$ ) may be triggered by peripheral input, whereas longer-duration activity requires, and is driven by, constant input.

shows that adjacent layer 4 electrodes (black) have a higher correlation coefficient than deeper electrodes (white) at the same locale. $\mathbf{G}$, Population (20 SATs from each of 3 pups) average of correlation coefficients. Electrode pairs were divided and averaged by horizontal (red or pink bars) or vertical (blue or green bars) separation distance for layer 4 (left) or layer $5 / 6$ (right). Layer 4 electrodes showed greater correlation with other layer 4 electrodes than with deeper electrodes regardless of separation distance. Layer $5 / 6$ electrodes showed less correlation than layer 4 electrodes regardless of separation or orientation. 


\section{Slow activity transients and the development of ongoing activity}

Two hypotheses have been put forward to account for the development of continuous activity in human neocortex: (1) a continuous elongation of spontaneous activity transients such as SATs or (2) a separate development of unrelated network activity, occurring during "intra-SAT" times and the independent elimination of SATs (Vanhatalo and Kaila, 2006). By showing two patterns of activity, each with its own generative mechanism and time course, during comparable developmental stages, our data support the later hypothesis. We observed a separate pattern of short bursts beginning at P8-P10 that was clearly different from rvSATs. Our data, and recent imaging studies (Golshani et al., 2009; Rochefort et al., 2009), have indicated that these bursts become more frequent and quickly transform into adult-like slow waves at the end of the second postnatal week, supporting the hypothesis that SATs and ongoing cortical activity are not related.

The relatively late development of slow waves is consistent with observations showing that spontaneous synaptic network activity in somatosensory cortical slices develops late in the first postnatal week in the form of cortical giant depolarizing potentials (Allène et al., 2008; Rheims et al., 2008). Surprisingly, given the prevalence of such activity in slices, in vivo such activity represented a small minority (13\%) of action potentials in cortex during the second postnatal week. Thus, although cortex, when isolated via enucleation, slicing, or anesthesia, can compensate rapidly by generating activity internally, it appears that under normal circumstances retinal waves provide a large majority of the neuronal activation in developing V1. Interestingly, we observed that clear internally generated activity in cortex does not occur until after the initial refinement of, and critical period for, the establishment of gross topography (Crowley and Katz, 2000; Olavarria and Hiroi, 2003; Cang et al., 2005). This may have important consequences for plasticity, because internal cortical activity may provide activation that retains formed (even misformed) connections even when the input is removed (Crowley and Katz, 1999).

\section{Physiological roles for SATs}

The ability of alpha and beta band oscillations to synchronize columnar activity in vivo (present paper and Yang et al., 2009) and in vitro (Dupont et al., 2006; Sun and Luhmann, 2007) has clear implications for synaptic plasticity mechanisms and circuit formation during cortical development. The firing of neighboring ganglion cells is poorly correlated by retinal bursts (Butts and Rokhsar, 2001). The thalamus and superior colliculus compensate for this with unique burst-based plasticity rules (Butts et al., 2007; Shah and Crair, 2008). Although such mechanisms have not been described in visual cortex, the beta oscillations we observe provide another avenue by which the poorly timed retinal inputs can drive cortical synaptic plasticity. Indeed, the timing of presynaptic cell firing relative to the phase of induced gamma oscillations determines the sign of synaptic plasticity in V1 (Wespatat et al., 2004). Furthermore, before eye opening, the sign of white matter $\rightarrow$ layer 4 synaptic plasticity is determined by the level of postsynaptic depolarization (Jiang et al., 2007). Thus, rvSATs and associated rapid oscillations may provide perfect conditions for bidirectional synaptic plasticity in the developing visual circuitry.

By showing in the present study that cortical oscillations are driven and not simply triggered by retinal input, we open the possibility that fine-scale information encoded in these waves can be transmitted to cortex. For example, during phase III retinal waves, nearby on and off ganglion cells become desynchronized (Kerschensteiner and Wong, 2008), a condition that modeling predicts is necessary for the development of orientation selectivity in cortex (Miller, 1994). Fine-scale propagation is consistent with the role of these waves in the refinement of retinal inputs to thalamus (Hooks and Chen, 2006). In summary, our results suggest that the developing thalamocortical circuit transmits retinal activity but transforms it into a network oscillation that may aid in the mechanisms of synaptic plasticity. We suggest that such transformations of peripheral input constitutes a general principle of activity transmission in cortex during early development.

\section{References}

Adelsberger H, Garaschuk O, Konnerth A (2005) Cortical calcium waves in resting newborn mice. Nat Neurosci 8:988-990.

Allène C, Cattani A, Ackman JB, Bonifazi P, Aniksztejn L, Ben-Ari Y, Cossart R (2008) Sequential generation of two distinct synapse-driven network patterns in developing neocortex. J Neurosci 28:12851-12863.

Ben-Ari Y, Gaiarsa JL, Tyzio R, Khazipov R (2007) GABA: a pioneer transmitter that excites immature neurons and generates primitive oscillations. Physiol Rev 87:1215-1284.

Blankenship AG, Ford KJ, Johnson J, Seal RP, Edwards RH, Copenhagen DR, Feller MB (2009) Synaptic and extrasynaptic factors governing glutamatergic retinal waves. Neuron 62:230-241.

Blumberg MS, Seelke AM, Lowen SB, Karlsson KA (2005) Dynamics of sleep-wake cyclicity in developing rats. Proc Natl Acad Sci USA 102:14860-14864.

Butts DA, Rokhsar DS (2001) The information content of spontaneous retinal waves. J Neurosci 21:961-973.

Butts DA, Kanold PO, Shatz CJ (2007) A burst-based "Hebbian" learning rule at retinogeniculate synapses links retinal waves to activity-dependent refinement. PLoS Biol 5:e61.

Cang J, Rentería RC, Kaneko M, Liu X, Copenhagen DR, Stryker MP (2005) Development of precise maps in visual cortex requires patterned spontaneous activity in the retina. Neuron 48:797-809.

Chiu C, Weliky M (2001) Spontaneous activity in developing ferret visual cortex in vivo. J Neurosci 21:8906-8914.

Chiu C, Weliky M (2002) Relationship of correlated spontaneous activity to functional ocular dominance columns in the developing visual cortex. Neuron 35:1123-1134.

Colonnese MT, Constantine-Paton M (2006) Developmental period for $N$-methyl-D-apartate (NMDA) receptor-dependent synapse elimination correlated with visuotopic map refinement. J Comp Neurol 494:738-751.

Crowley JC, Katz LC (1999) Development of ocular dominance columns in the absence of retinal input. Nat Neurosci 2:1125-1130.

Crowley JC, Katz LC (2000) Early development of ocular dominance columns. Science 290:1321-1324.

Dupont E, Hanganu IL, Kilb W, Hirsch S, Luhmann HJ (2006) Rapid developmental switch in the mechanisms driving early cortical columnar networks. Nature 439:79-83.

Garaschuk O, Linn J, Eilers J, Konnerth A (2000) Large-scale oscillatory calcium waves in the immature cortex. Nat Neurosci 3:452-459.

Golshani P, Gonçalves JT, Khoshkhoo S, Mostany R, Smirnakis S, PorteraCailliau C (2009) Internally mediated developmental desynchronization of neocortical network activity. J Neurosci 29:10890-10899.

Hanganu IL, Ben-Ari Y, Khazipov R (2006) Retinal waves trigger spindle bursts in the neonatal rat visual cortex. J Neurosci 26:6728-6736.

Hooks BM, Chen C (2006) Distinct roles for spontaneous and visual activity in remodeling of the retinogeniculate synapse. Neuron 52:281-291.

Huberman AD, Feller MB, Chapman B (2008) Mechanisms underlying development of visual maps and receptive fields. Annu Rev Neurosci 31:479-509.

Jiang B, Treviño M, Kirkwood A (2007) Sequential development of longterm potentiation and depression in different layers of the mouse visual cortex. J Neurosci 27:9648-9652.

Jouvet-Mounier D, Astic L, Lacote D (1970) Ontogenesis of the states of sleep in rat, cat, and guinea pig during the first post-natal month. Dev Psychobiol 2:216-239.

Kerschensteiner D, Wong ROL (2008) A precisely timed asynchronous pat- 
tern of ON and OFF retinal ganglion cell activity during propagation of retinal waves. Neuron 58:851-858.

Khazipov R, Luhmann HJ (2006) Early patterns of electrical activity in the developing cerebral cortex of humans and rodents. Trends Neurosci 29:414-418.

Khazipov R, Sirota A, Leinekugel X, Holmes GL, Ben-Ari Y, Buzsáki G (2004) Early motor activity drives spindle bursts in the developing somatosensory cortex. Nature 432:758 -761 .

Lamblin MD, André M, Challamel MJ, Curzi-Dascalova L, d'Allest AM, De Giovanni E, Moussalli-Salefranque F, Navelet Y, Plouin P, RadvanyiBouvet MF, Samson-Dollfus D, Vecchierini-Blineau MF (1999) Electroencephalography of the premature and term newborn. Maturational aspects and glossary (in French). Neurophysiol Clin 29:123-219.

Le Bon-Jego M, Yuste R (2007) Persistently active, pacemaker-like neurons in neocortex. Front Neurosci 1:123-129.

Lund RD (1965) Uncrossed visual pathways of hooded and albino rats. Science 149:1506-1507.

Marcano-Reik AJ, Blumberg MS (2008) The corpus callosum modulates spindle-burst activity within homotopic regions of somatosensory cortex in newborn rats. Eur J Neurosci 28:1457-1466.

Milh M, Kaminska A, Huon C, Lapillonne A, Ben-Ari Y, Khazipov R (2007) Rapid cortical oscillations and early motor activity in premature human neonate. Cereb Cortex 17:1582-1594.

Miller KD (1994) A model for the development of simple cell receptive fields and the ordered arrangement of orientation columns through activitydependent competition between ON- and OFF-center inputs. J Neurosci 14:409-441.

Minlebaev M, Ben-Ari Y, Khazipov R (2007) Network mechanisms of spindle-burst oscillations in the neonatal rat barrel cortex in vivo. J Neurophysiol 97:692-700.

Minlebaev M, Ben-Ari Y, Khazipov R (2009) NMDA receptors pattern early activity in the developing barrel cortex in vivo. Cereb Cortex 19:688-696.

Mizdorf U (1985) Current source-density method and application in cat cerebral cortex: investigation of evoked potentials and EEG phenomena. Physiol Rev 65:37-100.

Molnár Z, Kurotani T, Higashi S, Yamamoto N, Toyama K (2003) Development of functional thalamocortical synapses studied with current source-density analysis in whole forebrain slices in the rat. Brain Res Bull 60:355-371.

Moody WJ, Bosma MM (2005) Ion channel development, spontaneous activity, and activity-dependent development in nerve and muscle cells. Physiol Rev 85:883-941.

Mooney R, Penn AA, Gallego R, Shatz CJ (1996) Thalamic relay of spontaneous retinal activity prior to vision. Neuron 17:863-874.
Olavarria JF, Hiroi R (2003) Retinal influences specify cortico-cortical maps by postnatal day six in rats and mice. J Comp Neurol 459:156-172.

Prusky GT, Harker KT, Douglas RM, Whishaw IQ (2002) Variation in visual acuity within pigmented, and between pigmented and albino rat strains. Behav Brain Res 136:339-348.

Rheims S, Minlebaev M, Ivanov A, Represa A, Khazipov R, Holmes GL, Ben-Ari Y, Zilberter Y (2008) Excitatory GABA in rodent developing neocortex in vitro. J Neurophysiol 100:609-619.

Rochefort NL, Garaschuk O, Milos RI, Narushima M, Marandi N, Pichler B, Kovalchuk Y, Konnerth A (2009) Sparsification of neuronal activity in the visual cortex at eye-opening. Proc Natl Acad Sci U S A 106:1504915054 .

Shah RD, Crair MC (2008) Retinocollicular synapse maturation and plasticity are regulated by correlated retinal waves. J Neurosci 28:292-303.

Smith SL, Trachtenberg JT (2007) Experience-dependent binocular competition in the visual cortex begins at eye opening. Nat Neurosci 10:370375

Sun JJ, Luhmann HJ (2007) Spatio-temporal dynamics of oscillatory network activity in the neonatal mouse cerebral cortex. Eur J Neurosci 26:1995-2004.

Tolonen M, Palva JM, Andersson S, Vanhatalo S (2007) Development of the spontaneous activity transients and ongoing cortical activity in human preterm babies. Neuroscience 145:997-1006.

Vanhatalo S, Kaila K (2006) Development of neonatal EEG activity: from phenomenology to physiology. Semin Fetal Neonatal Med 11:471-478.

Vanhatalo S, Palva JM, Andersson S, Rivera C, Voipio J, Kaila K (2005) Slow endogenous activity transients and developmental expression of $\mathrm{K}^{+}-\mathrm{Cl}^{-}$ cotransporter 2 in the immature human cortex. Eur J Neurosci 22:27992804.

Warland DK, Huberman AD, Chalupa LM (2006) Dynamics of spontaneous activity in the fetal macaque retina during development of retinogeniculate pathways. J Neurosci 26:5190-5197.

Weliky M, Katz LC (1999) Correlational structure of spontaneous neuronal activity in the developing lateral geniculate nucleus in vivo. Science 285:599-604.

Wespatat V, Tennigkeit F, Singer W (2004) Phase sensitivity of synaptic modifications in oscillating cells of rat visual cortex. J Neurosci 24:90679075.

Yang JW, Hanganu-Opatz IL, Sun JJ, Luhmann HJ (2009) Three patterns of oscillatory activity differentially synchronize developing neocortical networks in vivo. J Neurosci 29:9011-9025.

Zhou Q, Tao HW, Poo MM (2003) Reversal and stabilization of synaptic modifications in a developing visual system. Science 300:1953-1957. 